\title{
PAST INTENSITY OF A TERMINATED POISSON PROCESS
}

\author{
S.M. POLLOCK
}

The University of Michigan, Ann Arbor, MI 48109, USA

\section{R.L. FARRELL \\ Vector Research, Incorporated}

Received October 1982

Revised June 1983

\begin{abstract}
A previously published analysis of the past behavior of a terminated stochastic process (Maltz and Pollock, 1980) is flawed. We present a correct analysis, specifically for the past intensity of a Poisson process stopped when the number of events within the past $w$ time units exceeds 2.
\end{abstract}

Stochastic processes, conditional stopping

\section{Introduction}

One rule for the termination of a stochastic point process is to stop the process when the number of events within a window of length $w$ exceeds a threshold number $k$. Such a behavior was hypothesized in [1] in an attempt to develop a model that might represent the decision-making of judges when facing a juvenile delinquent with a record of 'police contact' events extending into the past.

An interesting question arises when one wishes to look at the past behavior of a collection of processes terminated in this way. This was examined in [1] in an applied (criminal justice) setting, but unfortunately the mathematical analysis given there is flawed in not having taken into account the true conditional nature of the process before it is terminated. In this paper we present an analysis that rectifies this flaw. While the specific results of the paper are directed at a Poisson process and with stopping threshold $k=2$, they shed light on the types of behavior which may be expected in more general cases.

\section{Fundamental problem}

Consider a point process commencing at time 0 - that is, with no points in $(-\infty, 0]-$ and an associated stopping rule that terminates the process at the first time $t$ that there are $k$ events within the interval $[t-w, t]$. Let the stopping time be the random variable $T$, with density function $f(t)$. For example, if the process is Poisson with rate $\lambda$, then when $k=1, f(t)$ is trivially $\lambda \mathrm{e}^{-\lambda t}$. However, if $k \geqslant 2$, even in this simple case $(f(t)$ becomes an extremely complicated function (the case of $k=2$ is discussed in detail below).

We wish to find the rate of a stopped process $y$ time units into the 'past', e.g., previous to being stopped, but of course after the process started. The rate of interest is the conditional probability density of an event at the time $y$ in the past, given that the process was in existence at time $y$ in the past. That is, in looking back at time $y$ we consider only evidence from realizations which were 'alive' at that time; other realizations are disregarded.

This rate can be computed by first determining a rate conditioned on a particular value of the 
stopping time, defined to be

$\rho(y \mid t) \equiv$ prob. dens. \{nonstopping event at $t-y \mid$ process steps at $t\}$.

(Throughout this paper, we assume that the relevant densities exist, that functions are smooth, that limits taken exist, etc., without giving mathematical details or describing discontinuous analogs of the cases described.)

Once this has been computed, this allows us to find the unconditional rate:

$r(y)=\int_{0}^{\infty} \rho(y \mid t) h(y, t) \mathrm{d} t$

where

$h(y, t) \equiv$ p.d.f. for the stopping time $T$ given $T>y$

$$
= \begin{cases}0, & 0 \leqslant t \leqslant y, \\ \frac{f(t)}{\bar{F}(y)}, & y<t \leqslant \infty,\end{cases}
$$

with $\bar{F}(y)=\int_{y}^{\infty} f(t) \mathrm{d} t$.

\section{Solution for a Poisson process and $k=2$}

In the case where $k=2$ and the point process is a renewal process, equation (1) may be usually re-written by using Bayes' rule. In particular, by defining $g(y)=$ prob. dens. \{process stops at $t$ | nonstopping even at $t-y\}$ and $e(t-y)=$ prob. dens. \{nonstopping event at $t-y$ \} then equation (1) becomes

$\rho(y \mid t)=\frac{g(y) e(t-y)}{f(t)}$

Combining this with equations (2) and (3) gives, after some manipulation,

$r(y)=\frac{g(y) \int_{0}^{\infty} e(x) \mathrm{d} x}{\bar{F}(y)}$.

(This formula does not hold for $k>2$, in which case $\boldsymbol{g}$ is a function of $y$ and $t-y$.)

To find $g(y)$, we note that this is the p.d.f. for the time $Y$ from a (nonstopping) event to termination of the process. Simple renewal arguments lead to the generator equations:

$g(t)= \begin{cases}\lambda \mathrm{e}^{-\lambda t}, & 0 \leqslant t \leqslant w, \\ \int_{w}^{t} \lambda \mathrm{e}^{-\lambda x} g(t-x) \mathrm{d} x, & w<t .\end{cases}$
The upper expression on the right-hand side represents a termination at $t$ due to the next event's arrival time being less than $w$; the lower expression convolutes the exponential next-event time (larger than $w$ ) with $g(\cdot)$. Successive solutions of equation (5) for intervals $(w, 2 w],(2 w, 3 w], \ldots$ lead eventually to

$$
\begin{aligned}
g(y)= & \lambda \mathrm{e}^{-\lambda y}\left[\sum_{n=0}^{N} \frac{\lambda^{n}(y-n w)^{n}}{n !}\right. \\
& \left.-\sum_{n=0}^{N-1} \frac{\lambda^{n}(y-(n+1) w)^{n}}{n !}\right]
\end{aligned}
$$

where $N \equiv[y / w]=$ greatest integer part of $y / w$, and $\sum_{n=0}^{-1} \equiv 0$.

By similar arguments it can be shown that

$F(y)=\mathrm{e}^{-\lambda_{y} y} \sum_{n=0}^{N+1} \frac{\lambda^{n}[y-(n-1) w]^{n}}{n !}$.

By the additivity of expectations, $\int_{0}^{\infty} e(x) \mathrm{d} x$ is the expected number of nonstopping events. The number of such events is distributed geometrically with parameter $\mathrm{e}^{-\lambda w}$. Therefore

$\int_{0}^{\infty} e(x) \mathrm{d} x=\frac{1}{1-\mathrm{e}^{-\lambda w}}$.

Figure 1 shows $r(y) / \lambda$, as calculated from equation (4), using the results of equation (6), (7) and (8). In the figure $w=1$, so that time is in 'window units', an $r(y) / \lambda$ is shown for $=0.5,1,2$ and 5 . The figure illustrates a number of interesting features to this retrospective look at the terminated process, each of which can be proven

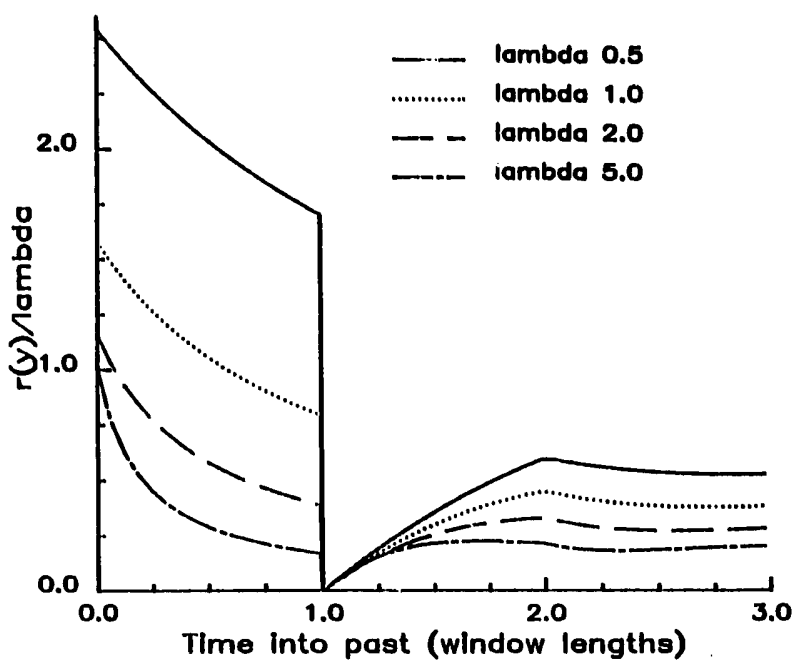

Fig. 1. Ratio of $r(y)$ to lambda. 
algebraically as well as observed in the cases shown in the figure:

(a) For all $\lambda$, the rate of occurrences into the past falls off until $t=1$ where it drips to zero.

(b) Values of $r>1 / w$ can occur even for $y>w$.

(c) For times greater than 1 into the past, although the rate climbs in some time periods, it is never greater than $\lambda$, and even for $\lambda<1$ can be considerably less than $\lambda$.

(d) As $\mathrm{y} \rightarrow \infty, r(y) / \lambda$ approaches a nonzero value dependent on $\lambda$ and $w$.

To analyze the limited behavior of $r(y)$ for large $y$, consider the defective renewal equations

$$
\begin{aligned}
& g(y)=b(y)+\int_{0}^{y} g(y-u) d L(u), \\
& F(y)=A(y)+\int_{0}^{y} F(y-u) d L(u),
\end{aligned}
$$

where

$$
\begin{aligned}
& A(y)= \begin{cases}1-\mathrm{e}^{-\lambda y}(1+\lambda y), & y<w, \\
1-\mathrm{e}^{-\lambda w}(1+\lambda w)+w\left(\mathrm{e}^{-\lambda w}-\mathrm{e}^{-\lambda x}\right), & y>w,\end{cases} \\
& b(y)= \begin{cases}\lambda \mathrm{e}^{-\lambda y}, & 0<y<w, \\
0, & y>w,\end{cases} \\
& L(y)= \begin{cases}0, & y<w, \\
\mathrm{e}^{-\lambda w}-\mathrm{e}^{-\lambda y}, & y>w .\end{cases}
\end{aligned}
$$

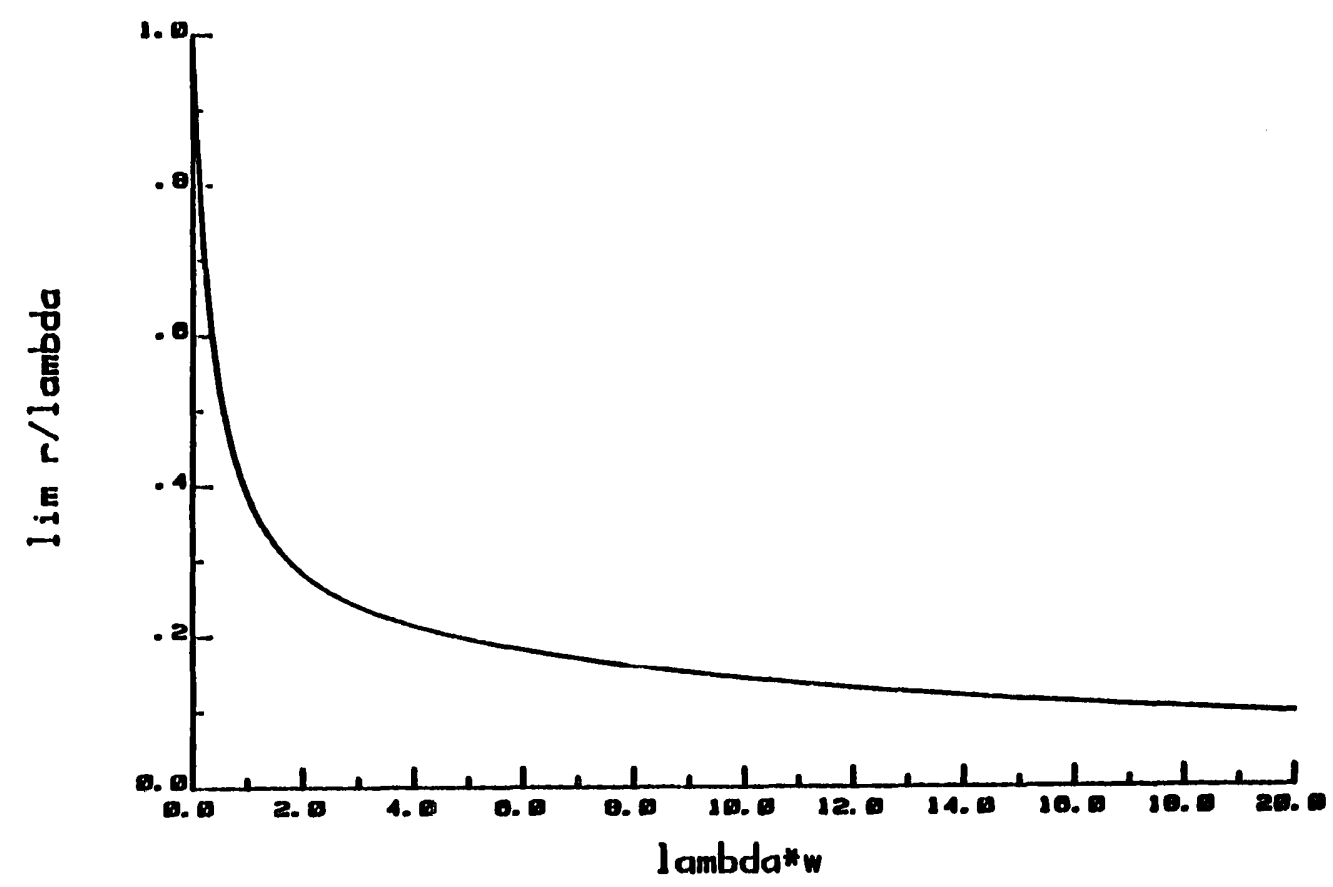

Fig. 2. Graph of lim $r /$ lambda.

\section{lambda*w}

Using methods for the asymptotic analysis of renewal equations with defective distributions (note that $\left.L(\infty)=\mathrm{e}^{-\lambda w^{\prime}}=1-P_{0}<1\right)$ given, for instance, in [2] and [3], one can eventually obtain

$$
\lim _{y \rightarrow \infty} r(y)=\lim _{y \rightarrow \infty} \frac{g(y) / P_{0}}{1-F(y)}=\frac{\lambda \eta(1-\eta)}{1-\mathrm{e}^{-\lambda w}}
$$

where $\eta$ is the (unique) solution to $\eta=\mathrm{e}^{-\eta w}$. Figure 2 shows $\lim _{y \rightarrow \infty} r(y) / \lambda$ as a function of $\lambda w$.

Analysis of even Poisson processes with $k>2$ has thus far proven to be intractable. The analytic difficulties are associated with the fact that the process does not regenerate at each event, and that an auxiliary state space of dimension $k-1$ is necessary to describe the state of the process at an event. A discrete-time approximation for general renewal cases with arbitrary $\boldsymbol{k}$ however, is possible - one that yields messy but computable results. The method for such an analysis is presented in [4].

\section{Acknowledgement}

We should point out that $\mathrm{L}$. Tierney brought to our attention the flaws in the development of $r(t)$ in [1]. Jeff Alden was particularly helpful in pointing out an elegant transform approach that allows a verification of equations (6) and (7). M. Maltz 
has developed a simulation of the process that confirms our results. Part of this research was funded by National Institute of Justice Grant \#81-IJ-CX 0064.

\section{References}

[1] M.D. Maltz and S.M. Pollock, "Artificial inflation of a delinquency rate by a selection artifact", Operations Research 28, 547-559 (1980).
[2] W. Feller, An Introduction to Probability Theory and Its Applications, Volume II, Wiley, New York, 1966.

[3] S. Karlin and H.M. Taylor, A First Course in Stochastic Processes, Second Edition, Academic Press, New York. 1975.

[4] S.M. Pollock and R.L. Farrell, "Past intensity of a terminated Poisson process", technical report, Department of of Industrial and Operations Research, University of Michigan, 1983. 